\title{
LB Lawremce Berkeley Laboratory
} UNIVERSITY OF CALIFORNIA

Submitted to Physical Review Letters

THE RISE AND FALL OF THE SPIN ALIGNMENT IN DEEP-INELASTIC REACTIONS

G.J. Wozniak, R.J. McDonald, A.J. Pacheco, C.C. Hsu,

D.J. Morrissey, L.G. Sobotka, L.G. Moretto, S. Shih,

C. Schück, R.M. Diamond, H. Kluge, and F.S. Stephens

July 1980

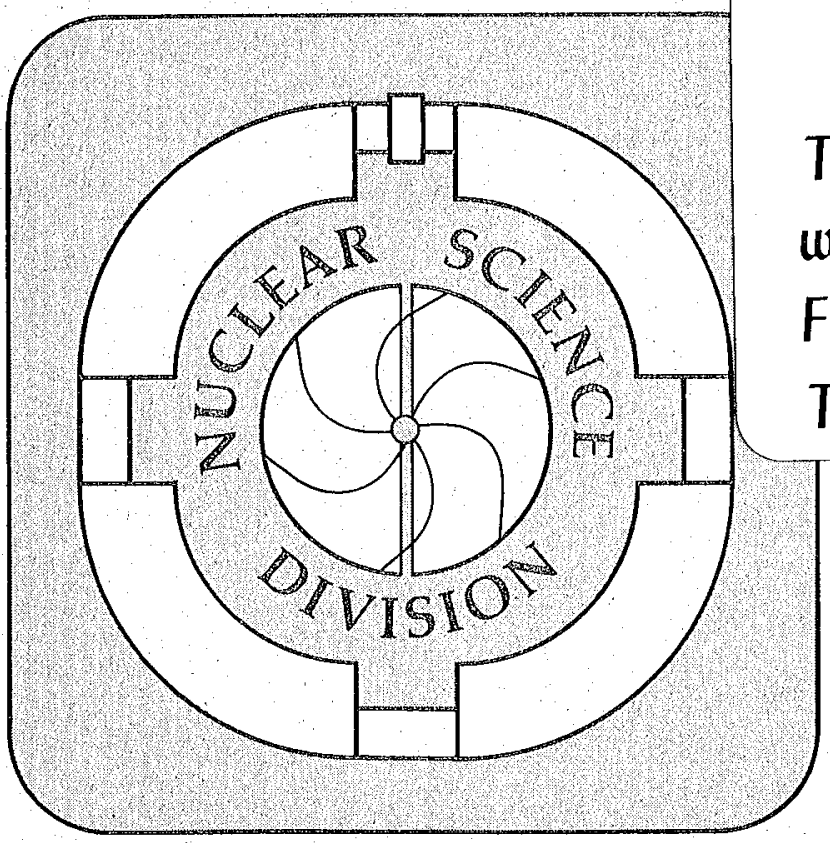

TWO-WEEK LOAN COPY

This is a Library Circulating Copy which may be borrowed for two weeks. For a personal retention copy, call Tech. Info. Division, Ext. 6782

Prepared for the U.S. Department of Energy under Contract W-7405-ENG-48 
THE RISE AND FALL OF THE SPIN ALIGNMENT IN DEEP-INELASTIC REACTIONS

G. J. Wozriak, R. J. McDonald, A. J. Pacheco, C. C. Hsu, (a)

D. J. Morrissey, L. G. Sobotka L. G. Moretto,

S. Shih, (b) C. Schüick, (c) R. M. Diamond,

H. Kluge, $(d)$ and F. S. Stephens

Nuc lear Science Division

Lawrence Berkeley Laboratory

University of $\mathrm{California}$

Berkeley, CA 94720

\section{ABSTRACT}

Both the magnitude and alignment of the transferred angular momentum in the reaction ${ }^{165} \mathrm{Ho}+{ }^{165}$ Ho have been measured as a function of Q-value via continuum $\gamma$-ray multiplicity and anisotropy techniques. Two regimes are observed: a low Q-value regime where the aligned angular momentum component dominates over the random components, and a large Q-value regime where the random components dominate and substantially decrease the spin alignment.

\footnotetext{
(a) Permanent address: Institut of Atomic Energy, Beijing, China.

(b) Permanent address: Shanghai Institute of Nuclear Research, Shanghai, China.

(c) Permanent address: C. S. N. S. M., Orsay, France.

(d) Permanent address: Hahn-Meitner Institute, Berlin, Germany.
} 
Angular momentum is known to be transferred from orbital to intrinsic degrees of freedom in heavy-ion collisions. Information regarding the mechanism of this process can be obtained from determinations of the magnitude and alignment of the fragment spin as a function of Q-value. The correlation of spin transfer with energy damping has been investigated with both $\gamma$-ray multiplicity ${ }^{1-3}\left(M_{\gamma}\right)$ and sequential-fission techniques. ${ }^{4,5}$ These studies have shown that the angular-momentum transfer increases with increasing Q-value until it saturates in the deep-inelastic region. For both light and heavy systems, anomalously large second moments ${ }^{1,2,6}$ of the $r$-ray multiplicity distributions have been observed as well as small continuum $\gamma-$ ray anisotropies. ${ }^{6-9}$ These data have been interpreted as evidence for the presence of random spin fluctuations. ${ }^{9,10}$ Although spin alignment has been observed with discrete lines, ${ }^{11,12}$ doubts have been expressed regarding the feasibility of using continum $\gamma$-rays to study either the spin transfer or alignment in deep-inelastic (DI) reactions. However, if one chooses a system where the random spin fluctuations can be made to vary from small to large relative to the magnitude of the aligned component, one may observe large corresponding changes in the continuum $\gamma$-ray anisotropy.

In this Letter we report the simultaneous measurement of the magnitude and alignment of the angular-momentum transfer in the 8.5 MeV/A ${ }^{165} \mathrm{Ho}+{ }^{165} \mathrm{Ho}$ reaction as a function of Q-value via continuum $\gamma$-ray multiplicity and anisotropy techniques. This system was chosen because large amounts of angular momentum can be transferred 
into the intrinsic spin (I) of these nuclei, which are known to have good rotational properties. ${ }^{13,14}$ Furthermore, the steep massas ymmetry potential causes the reaction products to lie within a narrow range of $Z$-values centered around symmetry (as verified with a $\Delta E-E$ telescope) and minimizes e-fractionation effects. ${ }^{15}$ As a consequence, both of the essentially identical DI-fragments emit similar continuum $r-r$ ay spectra which are strongly enriched in E2 transitions $(\sim 80 \%)$ as discussed below.

A schematic diagram of the experimental setup is shown as an insert in Fig. 1. A beam of $8.5 \mathrm{MeV} / \mathrm{A}{ }^{165} \mathrm{Ho}$ ions from the Lawrence Berkeley Laboratory SuperHILAC bombarded a $0.85 \mathrm{mg} / \mathrm{cm}^{2}$ self-supporting ${ }^{165}$ Ho target. Two silicon E-detectors with $4.4^{\circ}$ acceptance angles were placed $27^{\circ}$ from the beam axis in two perpendicular planes. In each of these planes a $12.7 \mathrm{~cm}$ diameter by $15.7 \mathrm{~cm}$ deep $\mathrm{NaI}$ detector was placed at $90^{\circ}$ to the beam axis and at $60 \mathrm{~cm}$ from the target. This distance was sufficient to separate the $\gamma$-rays from neutrons by their time-offlight. A $5 \mathrm{~cm}$ thick $\mathrm{Pb}$ collimator having a $8.2 \mathrm{~cm}$ diameter tapered opening was utilized both to enhance the photopeak to Compton yield and to restrict the solid angle in order to limit prompt $\gamma-\gamma$ summing to $<5 \%$. The energy dependence of the absolute efficiency of the $\mathrm{NaI}$ counters between 0.3 and $1.8 \mathrm{MeV}$ was determined with coincident $\gamma$-ray cascade transitions.

Figure 1(a) illustrates the general features of both the in-plane and out-of-plane $\gamma$-ray spectra emitted from the reaction fragments. 
The spectral shapes are similar to those observed in compound-nucleus reactions 13,14 and display the characteristic "E2 bump" at 0.6-1.2 MeV and the higher energy "statistical tail" (2-5 MeV). A comparison of these two spectra indicates that the tail region is nearly isotropic, as seen in compound-nuc leus reactions, whereas the "bump" region is more pronounced in plane where the angular distribution for stretched E2 $\operatorname{tr}$ ansitions peaks.

In-plane $\gamma$-ray energy spectra, normalized so that the integral of each curve is equal to $\left\langle M_{\gamma}\right\rangle$, are shown in Fig. 1 (b) for several Qvalue regions. The upper edge of the "bump" moves to higher $\gamma$-ray energies as $Q$ increases in the quasi-elastic (QE) region and stabilizes in the DI-region. This is an indication of the Q-value dependence of the angular momentum transfer, since for rotational nuclei $E_{\gamma} \propto I$.

The angle-integrated $\gamma$-ray multiplicity was calculated from

$$
\left\langle M_{\gamma}\right\rangle=N_{\gamma} /\left[W\left(90^{\circ}\right) N_{\text {singles }}\right]
$$

where $N_{\gamma}$ is the efficiency-corrected number of coincident $\gamma$-rays. The angular-distribution function, $W(\theta)$, is normalized such that $\int W(\theta) d \Omega=4 \pi$, and $N_{\text {singles }}$ is the number of particle singles. The fragment spin after particle emission was calculated from

$$
\langle I\rangle=\frac{1}{2}\left(I_{1}+I_{2}\right)=\left\langle M_{\gamma}\right\rangle+2 H-2 B
$$

where $\mathrm{H}$ is the number of $\mathrm{E2}$ transitions below the $0.3 \mathrm{MeV}$ threshold (set to exclude the backscatter region) and $B$ is the number of 
statistical transitions per fragment. We assumed that the statistical transitions carry away no angular momentum on the average, and selected the $v$ alue $H=3$ after inspecting the $r$-decay schemes of even- and odd-mass products between $A=165$ and $A=150$.

Figure $2(a)$ shows the fragments' energy spectrum obtained at an angle slightly greater than the grazing angle. Figure 2(b) shows the intrinsic spin of one of the two reaction fragments after neutron emission (circles) as calculated from Eq. (2). The primary fragment spin obtained from $\left\langle M_{\gamma}\right\rangle$ with correction for neutron emission ${ }^{16}$ (solid line) is also shown. As seen in the data, the transferred spin rapidly increases with Q-value in the QE-region and saturates at about $35 \mathrm{~h}$ in the DI-region.

The ratio of in-plane to out-of-plane $\gamma$-ray yield ("anisotropy") for energies between 0.6 and $1.2 \mathrm{MeV}$ (squares) is also shown in Fig. 2(b). This anisotropy rises with increasing spin transfer; it peaks at a value of $\sim 2.2$, slightly before the spin saturates, and then drops to near unity for large Q-values. Figure $2(c)$ shows the dependence of the anisotropy on both $E_{\gamma}$ and $Q$ in a two-dimensional contour diagram. One notes that the highest anisotropy occurs in the 0.6 to 1.2 MeV $E_{\gamma}$ region, where the "E2 bump" is most prominent, and at intermediate Q-values. At higher Q-Values, the anisotropy peaks at higher $E_{\gamma}$. These trends are consistent with the rotational nature of these $\gamma$-ray spectra. 
The initial rise of anisotropy with increasing Q-value indicates that during the early stages of energy damping there is a rapid buildup of aligned spin. The subsequent fall observed at larger Q-values suggests that the aligned component of spin has saturated or is decreasing, whereas randomly-oriented components continue to increase, causing a significant decrease in the alignment of the fragments' spin. These qualitative features are similar to those observed with discrete lines from the ${ }^{16} 0+{ }^{48} \mathrm{Ti}$ system. ${ }^{12}$ However, in this much heavier system, the rise and fall of the alignment is observed over a substantially larger Q-value range with continuum $r$-rays. This buildup of alignment has also been seen in sequential-fission studies, 5 but a decrease was not observed in the more limited Q-value range studied. Interpretation of these data may be approached in several ways, e.g., dynamical models, non-equilibrium statistical mechanics, etc. We choose to utilize the statistical equilibrium model of Moretto and Schmitt ${ }^{17,18}$ which applies to the long-time thermal limit toward which all other models should tend. This model describes a primary depolarization mechanism which is the thermal excitation of angular momentum bearing collective modes ${ }^{17}$ (wriggling, tilting, bending, and twisting) in a rotating di-nuclear system of equal touching spheres. The resulting distribution for the Cartesian components of the angular momentum is Gaussian:

$$
P\left(I_{x}, I_{y}, I_{z}\right) \propto \exp \left[-\frac{I_{x}^{2}}{2 \sigma_{x}^{2}}-\frac{I_{y}^{2}}{2 \sigma_{y}^{2}}-\frac{\left(I_{z}-\bar{I}_{z}\right)^{2}}{2 \sigma_{z}^{2}}\right]
$$


and for this model:

$$
\sigma_{x}^{2} \cong \sigma_{y}^{2} \cong \sigma_{z}^{2} \equiv \sigma^{2}=1 T
$$

where is the moment of inertia for one of the spherical nuclei and $T$ is the temperature. This distribution function has been used to derive an expression for the $\gamma-r$ ay angular distribution ${ }^{19}$ in terms of $\sigma / \bar{I}_{z}$. Corrections have been made ${ }^{16}$ for contributions from the secondary depolarization process of neutron evaporation which precedes the $\gamma$-ray cascade.

Figure 3(a) shows experimental values of the anisotropy for $E_{\gamma}$ greater than $0.3 \mathrm{MeV}$ compared to several stages of the model calculation. The number of statistical transitions per fragment, $B$, was estimated from the shape of the $\gamma$-ray spectra for each Q-value bin assuming that the intensity of the statistical transitions is described by: ${ }^{14} P\left(E_{\gamma}\right) \propto E_{\gamma}^{2} \exp \left(-E_{\gamma} / T\right)$, where $T=0.6 \mathrm{MeV}$. With this value for $B$, the measured $\left\langle M_{\gamma}\right\rangle$, and $H=3$, the spin $\langle I\rangle$ was determined from Eq. (2), and the anisotropy was then calculated (Fig. 3(a)), solid line). This calculation reproduces both the shape and the magnitude of the data. To give a feeling for the importance of various contributions, the same calculation is shown with no correction for neutron evaporation (curve 2), assuming no statistical transitions (curve 3), and with no thermal effects (curve 4). This 
comparison clearly shows that the most important effect is the thermally induced misalignment, indicating that the decrease of alignment as deduced from the anisotropy is inherent to the deep-inelastic process itself. The misalignment due to statistical $\gamma$-ray emission is negligible $\left(\sigma^{2} \sim 3 \hbar^{2}\right)$.

By gating on the 0.6-1.2 MeV region of the $E_{\gamma}$ spectra, one both increases the fraction of E2-transitions and biases the spin distribution to larger values $\left(E_{\gamma} \propto I\right)$, which should yield larger anisotropies. In Fig. 3(b), measured (symbols) and calculated (solid line) anisotropies are shown for the 0.6-1.2 MeV $\gamma$-ray region. These data show the expected larger arisotropies, which the model calculations reproduce. The model prediction (corresponding to the solid line) of the a Tignment parameter $P_{Z Z}=\left(3\left\langle I_{z}^{2}\right\rangle \cdot 12\left\langle I^{2}\right\rangle-1 / 2\right)$ before neutron emission is 0.74 for the Q-value associated with the largest anisotropy and decreases to 0.58 for the largest Q-value.

The above $\gamma$-ray multiplicity and anisotropy data in conjunction with the model calculations give rise to the following picture of the reaction process. In the QE-region, the transferred spin increases rapidily with Q-value whereas the thermally misaligned components increase more slowly $\left(T \propto|Q|^{1 / 2}\right)$. Thus, the aligned component dominates and the transferred angular momentum is nearly perpendicular to the reaction plane, giving a large anisotropy. However, across the DI-region the transferred angular momentum saturates while the thermal components increase to become an ever larger fraction of the totâl angular momentum. Thus, the alignment decreases and the anisotropy plummets. 


\section{ACKNOWLEDGMENT}

This work was supported by the Nuclear Science Division of the

U. S. Department of Energy under Contract No. W-7405-ENG-48.

\section{REFERENCES}

1) P. R. Christensen. et a1., Phys. Rev. Lett. 40, 1245 (1978).

2) A. 01mi, et al., Phys. Rev. Lett. 41,688 (1978).

3) R. Regimbart, et al., Phys. Rev. Lett. 41, 1355 (1978).

4) D. v. Harrach, et al., Phys. Rev. Lett 42, 1728 (1978).

5) R. J. Puigh, et al., Phys. Lett. 86B, 24 (1979).

6) R. A. Dayras, et a1., Phys. Rev. Lett. 42, 697 (1979).

7) M. Berlanger, et al., J. de Phys. Lett. 37, L323 (1976).

8) J. B. Natowitz, et al., Phys. Rev. Lett. 40, 751 (1978).

9) P. Aguer, et a7., Phys. Rev. Lett. 43, 1778 (1979).

10) G. J. Wozniak, et al., Phys. Rev. Lett. 40, 1436 (1978).

11) R. J. Puigh et al., Nucl. Phys. A336, 279 (1980).

12) H. Puchta, et a1., Phys. Rev. Lett. 43, 623 (1979).

13) M. A. Deleplanque, et al., Phys. Rev. Lett. 41, 1105 (1978).

14) F. S. Stephens, Lawrence Berkeley Laboratory Report No., 10356 , published in the Proc. of the Varenna Conf., July 1979.

15) M. Aleonard, et a1., Phys. Rev. Lett. $\underline{40}, 622$ (1978).

16) S. K. Blau and L. G. Moretto, Lawrence Berkeley Laboratory Report No., LBL-10926 (to be published).

17) L. G. Moretto and R. P. Schmitt, Phys. Rev. C21, 204 (1980).

18) L. G. Moretto, Lawrence Berkeley Laboratory Report No., LBL-10463, published in the Proc. of the XVII Intern. Winter Meeting on Nuclear Phys. Bormio, Italy (1980). 
19) L. G. Moretto, S. Blau, and A. J. Pacheco, Lawrence Berkeley Laboratory Report No. LBL-10805 (to be published). 


\section{FIGURE CAPTIONS}

Fig. 1. (a) In- and out-of-plane $\gamma-r$ ay pulse-height spectra associated with reaction products having a Q-value of -140 MeV. Data points are shown only for the in-plane spectrum. Detectors $\mathrm{NaI}(1)$ and $\mathrm{Si}(1)$ are in the same plane.

(b) In-plane $\gamma$-ray pulse-height spectra for representative Q-value bins.

Fig. 2. (a) Particle spectrum for the ${ }^{165}$ Ho $+{ }^{165}$ Ho reaction at $27^{\circ}$ in the laboratory. (b) Post- (circles) and pre- (solid line), neutron emission values of the spin per fragment $\left\langle I_{1}\right\rangle$ as a function of the reaction Q-value (see text). The anisotropies, $W(i n / 1)$, extracted from the raw $N a I$ spectra are also shown (squares) for the $E_{\gamma}$ region 0.6-1.2 MeV. (The raw data and the unfolded spectra gave the same anisotropies to 5\%.) (c) Anisotropy contours for coordinates $E_{\gamma}$ and $Q$.

Fig. 3. Experimental (symbols) and calculated (curves) values of the anisotropy, $W(\mathrm{in} / 1)$, (a) for $\gamma$-rays greater than $0.3 \mathrm{MeV}$ and (b) for the region $0.6 \mathrm{MeV}<E_{\gamma}<1.2 \mathrm{MeV}$ (see "text). 


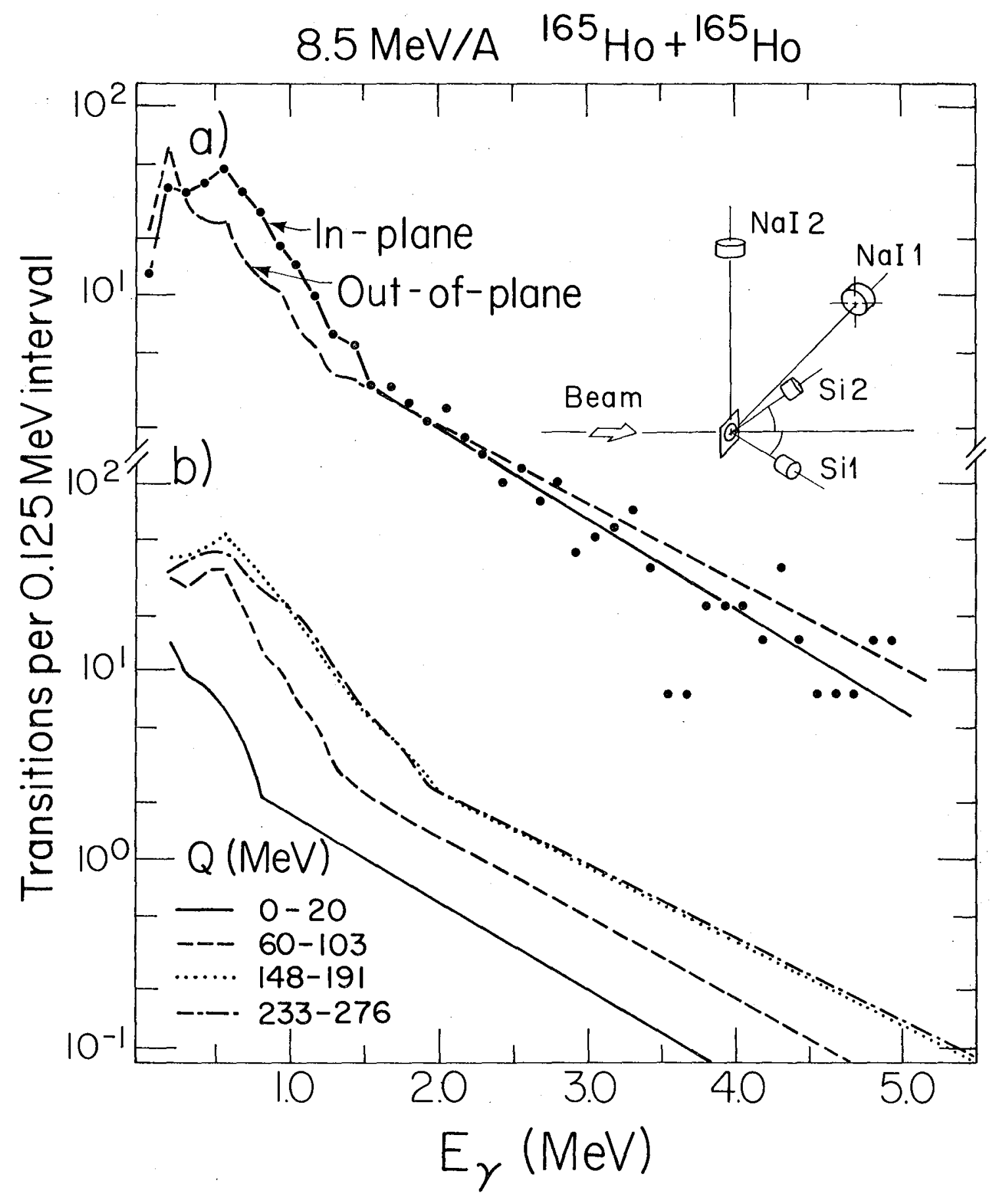

Fig. 1

XBL 806-1194 


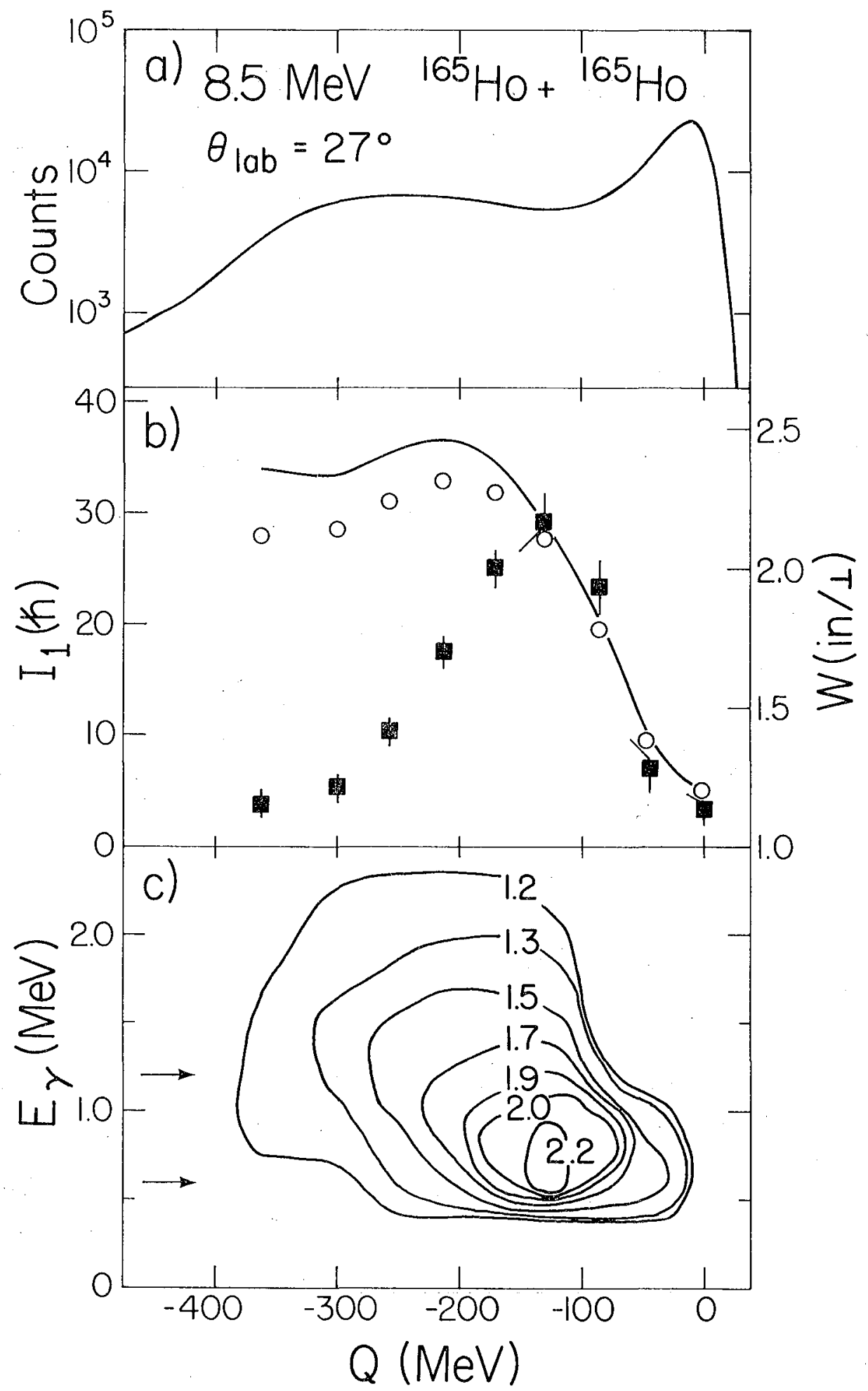

Fig. 2

$X B L 806-1214$ 


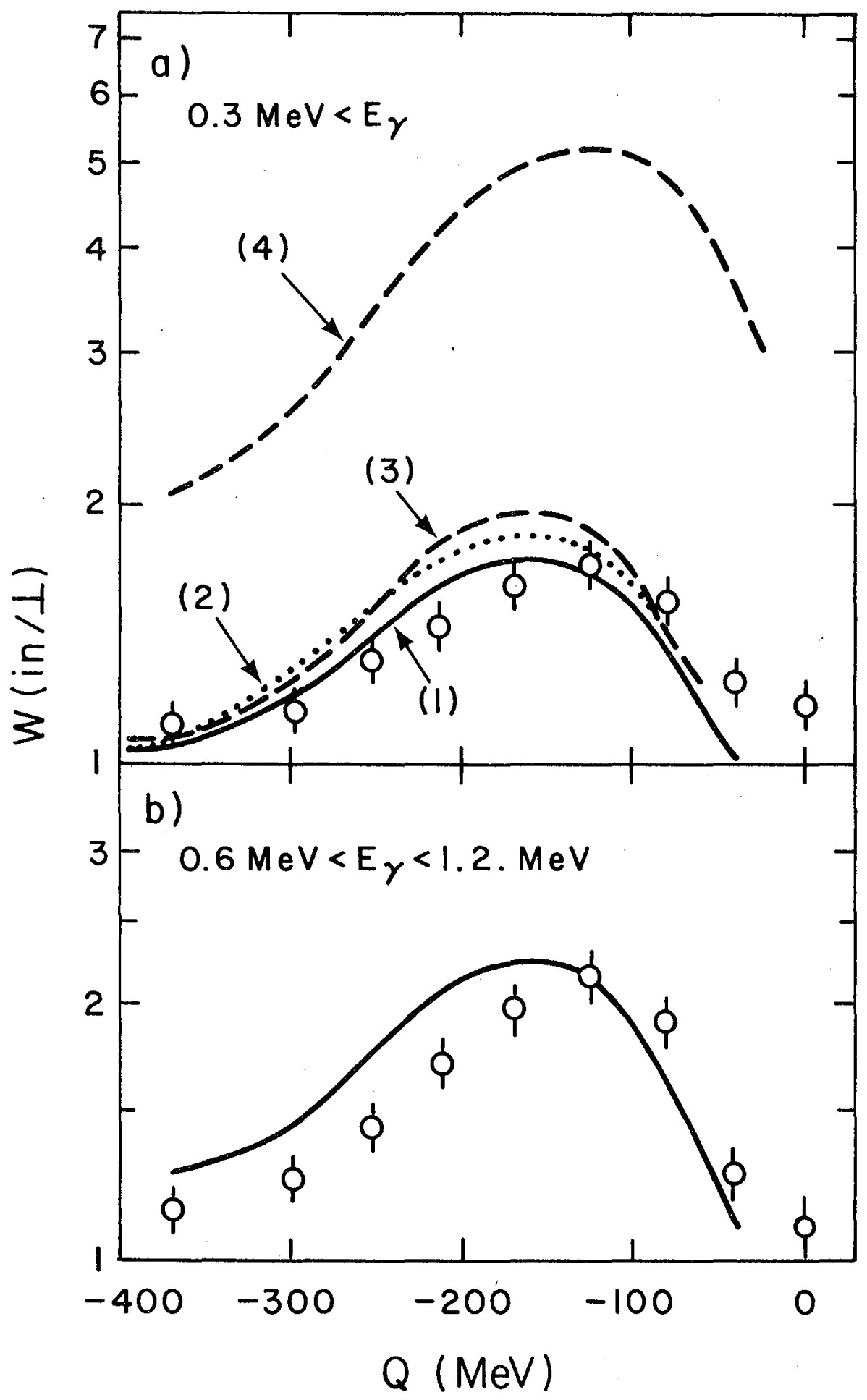

Fig. 3

XBL806-1193 
This report was done with support from the United States Energy Research and Development Administration. Any conclusions or opinions expressed in this report represent solely those of the author(s) and not necessarily those of The Regents of the University of California, the Lawrence Berkeley Laboratory or the United States Energy Research and Development Administration. 